\title{
Wakefield suppression in high gradient linacs for lepton linear colliders
}

\author{
Roger M. Jones ${ }^{1,2, *}$ \\ ${ }^{1}$ School of Physics and Astronomy, The University of Manchester, Oxford Road, Manchester, M13 9PL, United Kingdom \\ ${ }^{2}$ Cockcroft Institute of Science and Technology, Daresbury, WA4 4AD, United Kingdom
}

(Received 22 June 2009; published 8 October 2009)

\begin{abstract}
The transverse wakefield excited by multiple bunches in ultrarelativistic charged particle beams in a linear collider must be adequately damped in order to preserve the luminosity of the colliding beams and to prevent a beam breakup instability developing. A review of the means of achieving this wakefield suppression is discussed in detail for normal conducting linacs utilized for linear colliders. In particular, the features of a damped and detuned series of structures are described and compared to other methods. A conspectus of experimental results along with theoretical predictions for this series of accelerating structures developed for the next-linear-collider/global-linear-collider is presented together with the prospects for wakefield suppression for the compact linear collider.
\end{abstract}

DOI: 10.1103/PhysRevSTAB.12.104801

PACS numbers: 29.20.Ej, 41.75.Ht

\section{INTRODUCTION}

It is generally agreed within the particle physics community that the successor to the LHC [1,2] will be an electron-positron linear collider. The baseline design for linacs in the international linear collider (ILC $[3,4]$ ) utilizes superconducting (SC) technology and aims at a gradient of $31.5 \mathrm{MV} / \mathrm{m}$ along a linac which is approximately $10 \mathrm{~km}$ long. Each SC cavity, based on the TESLA technology choice [5], consists of nine cells and, depending on the location within the linac, there are 8 or 12 of these cavities within a given SC module. A train of 2625 bunches of charged particles will be accelerated up to an energy of $250 \mathrm{GeV}$. There are also alternate designs which aim at a gradient of $50 \mathrm{MV} / \mathrm{m}$. However, other than single cells [57] demonstrating the technique, no cavities have reached this ambitious gradient. In order to attain high gradients, it is generally agreed that normal conducting (NC), room temperature accelerators are necessary. This review paper is focused on high gradient $\mathrm{NC}$ accelerators.

There were two main NC linear collider research efforts up until 2004 which were concentrated on the next linear collider/global linear collider (NLC/GLC $[8,9]$ ) design and on the compact linear collider (CLIC) design [10-12]. After careful consideration, the International Technical Recommendation Panel (ITRP) decided to consolidate the efforts of the SC TESLA and NC NLC/GLC research efforts into a single SC technology choice. The CLIC NC design however remains an option to reach high center of mass energies and this is achievable through the larger gradient achievable in $\mathrm{NC}$ accelerator structures. The

\footnotetext{
*School of Physics and Astronomy, The University of Manchester, Oxford Road, Manchester, M13 9PL, United Kingdom.

roger.jones@manchester.ac.uk
}

present baseline design for CLIC incorporates a center of mass energy of $3 \mathrm{TeV}$ with a loaded accelerating gradient of $100 \mathrm{MV} / \mathrm{m}$ (although there is also a design for an electron-positron collision scheme for CLIC at $500 \mathrm{GeV}$ ). By necessity, NC accelerators for linear colliders must operate in a short pulse mode, otherwise Ohmic losses in the walls of the cavity will result in a prohibitively low overall efficiency. Whereas for SC accelerators, a long pulse train is acceptable as there are minimal $\mathrm{rf}$ losses. The ILC bunch train is approximately $1 \mathrm{~ms}$ long and the CLIC bunch train is markedly shorter at $156 \mathrm{~ns}$. The fundamental difference of the CLIC scheme from the ILC lays in the dual-beam method used in CLIC. The drive beam operates at a high current of $100 \mathrm{~A}$ and is decelerated in order to serve as a source of $\mathrm{rf}$ power to the main accelerating beam which operates at a current of $1 \mathrm{~A}$. The reason for utilizing a two-beam scheme design comes from the necessity of reducing the number of rf klystrons to a manageable number in a $3 \mathrm{TeV}$ lepton collider. However, a single beam scheme, as was designed for the NLC/GLC is possible for lower center of mass energies such as $500 \mathrm{GeV}$ and $1 \mathrm{TeV}$. The detailed components and parameters in the CLIC scheme are discussed in [10]. We confine this work to the electromagnetic (e.m.) field excited in the cavities of each of the main accelerating linacs, each of which is $21.1 \mathrm{~km}$ long and will contain 71406 structures [13]. The wealth of knowledge gained in more than one and a half decades of research on the NLC/GLC is indeed valuable for CLIC as rf breakdown and wakefields suppression issues, for example, are common concerns for both NLC/ GLC and CLIC.

In general, the introduction of a charged particle bunch into a cavity excites an e.m. field which acts both on the bunch itself and on successive bunches in the train. This e.m. field is conveniently represented as a wakefield 
$[14,15]$, in which we refer to that acting along the bunch as the short range wakefield and that further along the train as the long-range wakefield. Furthermore, the wakefield can be decomposed into that acting both longitudinally and transverse to the acceleration axis. The former modifies the energy of the bunches and can be controlled by Balakin, Novokhatsky, and Smirnov [16] damping and the latter modifies the emittance of the bunches and can cause a severe beam break up instability [17]. In this work we focus on means of suppressing the transverse long-range wakefield. The transverse wakefield is more severe for the accelerating structures designed for CLIC as the average iris radius is considerably smaller than that in the ILC. Indeed, it can be shown $[14,18]$ that in the diffraction limit valid for short bunches the envelope of the monopole (dipole) longitudinal (transverse) wakefield is inversely proportional to $a^{1.7}\left(a^{2.7}\right)$, where $a$ is the average cell radius of an accelerating cavity. In general, longitudinal and transverse wakes, from geometrical scaling considerations, can also be shown to be proportional to frequency as $\omega^{2}$ and $\omega^{3}$, respectively. The average iris radius of cells in the ILC cavities is approximately $35 \mathrm{~mm}$ and this compares with $3 \mathrm{~mm}$ for CLIC cavities. The CLIC accelerating frequency is $12 \mathrm{GHz}$ and this is almost a factor of 10 larger than the ILC monopole frequency of $1.3 \mathrm{GHz}$. The CLIC accelerating structure $\mathrm{X}$-band frequency is the outcome of a careful optimization strategy [19] based on maximizing the beam luminosity while minimizing the cost and at the same time ensuring rf breakdown issues, amongst other constraints, are adhered to. As the wakefield is more severe for X-band structures, the main focus of this paper will be on means of suppressing the wakefield in CLIC and related NLC/GLC structures at X-band frequencies (although Cband and S-band NC accelerators have also shown considerable promise as potential candidates for next generation high energy colliders and will also be discussed to a limited extent).

This review paper is organized such that the next main section discusses the means of wakefield suppression in $\mathrm{NC}$ accelerating structures. The penultimate section is concerned with an analysis of the beam dynamics in electron-positron colliders for $\mathrm{NC}$ accelerating structures. The final main section presents a discussion of future prospects for wakefield suppression and some conclusions on the present work.

\section{WAKEFIELD SUPPRESSION FOR NC STRUCTURES}

There are several possible methods available to suppress transverse wakefields excited in multicell cavities of a NC linear collider. However, it is important that in suppressing the parasitic modes excited by the beam the accelerating mode is left largely unaffected. There are several approaches available to affect this wakefield suppression. These methods can be grouped in two main schemes: heavy damping (which entails $Q$ values as low as $\sim 10$ ) and moderate damping (with $Q$ values $\sim 300-1000$ ). The former method is the baseline design for the CLIC main linac accelerating cavities and entails waveguidelike structures directly impinging into each accelerating cell. The latter method relies on heavy detuning of the cell frequencies and often takes advantage of the natural partitioning of modes into characteristic passbands (in the structures with a $2 \pi / 3$ per cell phase advance the modes which dominate the transverse momentum kick are located in the first passband [20,21]) and hence only a limited number of bands need to be damped.

Prior to discussing the major design strategies of wakefield suppression, the means of measuring the wakefield are discussed.

\section{A. Wakefield measurement}

In all long-range wakefield suppression schemes it is important to be able to verify the efficacy of the method employed and indeed to ascertain the accuracy of the simulation tools used. The transverse wakefield can be measured by several means. The most direct method entails ascertaining the transverse momentum kick imparted to trailing bunches. Beam-based measurement is necessarily expensive and requires high precision measurement techniques. Nonetheless, at the SLAC National Accelerator Laboratory there existed a facility, known as ASSET [22], which provided just such a measurement system, based on a positron excitation bunch and a measurement of the kick imparted to a trailing electron, or witness, bunch. Now as electron or positron bunches rapidly become ultrarelativistic (i.e. essentially traveling at the speed of light), the e.m. field is transverse to the direction of motion and is a TEM-like e.m. wave. For this reason, bench measurements, which simulate a charged particle beam using a wire offset from the center of an accelerating cavity, can be used to measure the beam impedance. This stretched wire measurement technique was originally proposed by Sands and Rees [23]. Taking the Fourier transform of beam impedance enables the wakefield to be recovered. However, it is often the case that a large fraction of the field at a particular frequency is located in a small number of cells of the accelerator cavity. These are known as trapped modes and are often difficult to measure by wire based techniques [24-26]. The beam-based measurement does not suffer from these inadequacies. A short description of the method used at the SLAC National Accelerator Laboratory is now provided.

The wakefield measurement using the accelerator structure setup (ASSET) facility in sector 2 of the $3 \mathrm{~km}$ long linac at SLAC is shown schematically in Fig. 1. This consists of a drive positron bunch extracted from the south damping ring which excites a wake and a witness electron bunch extracted from the north damping ring which is used to monitor the transverse momentum kick. During experi- 


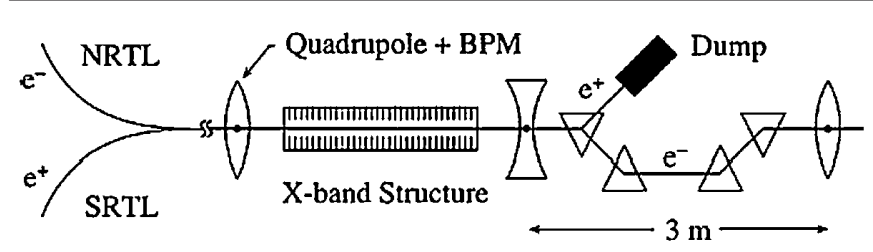

FIG. 1. Schematic representation of ASSET facility at SLAC National Accelerator Laboratory.

ments, the accelerating structure under test or the device under test (DUT) is maintained at its design operating temperature of $45^{\circ} \mathrm{C}$. The fundamental mode input and output couplers are terminated, either through similar cable connections or a matched load. In the linac, the driving bunch passes through the DUT and subsequently steered into a beam dump to dispense with the spent beam. The magnet used for this purpose is also the first bend of a chicane which transports electrons back onto the linac axis. The electron bunch serves as the witness bunch, and is extracted from the north damping ring at a later time and injected on-axis into the linac via the north-ring-to-linac transport line. In traversing the DUT, the witness bunch is deflected by the wakefield generated by the drive bunch. The witness bunch then passes though the chicane and down the linac where its trajectory is recorded by several beam position monitors located in each of the focusing quadruple magnets. The transverse wakefield is determined by measuring the change in the witness bunch deflection per unit charge in the drive bunch offset in the structure.

The angular kick imparted to the witness bunch can be obtained from considering the ratio of the transverse to longitudinal energy:

$$
\Delta \theta_{y}=\zeta W_{\perp}(t) \Delta y_{d} / E_{w},
$$

where $W_{\perp}(t)$ is the transverse wakefield at time $t$ behind the drive bunch, $E_{w}(=1.2 \mathrm{GeV})$ is the witness bunch energy, and $\Delta y_{d}$ is the offset in the drive bunch from the electrical center of the accelerating structure. The wakefield is provided with units of the transverse voltage imparted divided by the product of the total drive charge $\left(e n_{d}\right)$, drive offset, and structure length $\left(L_{s}\right)$. In practice, the charged particle bunches are assumed to be Gaussian and the factor $\zeta$ is given by

$$
\zeta=e^{2} L n_{d} \exp \left(-\omega^{2} \sigma^{2} / c^{2}\right) .
$$

Here $L$ is the length of the structure, $n_{d}$ is the total number of particles in the drive charge bunch, $\omega / 2 \pi$ is the central frequency of the distribution, and $\sigma$ is the rms bunch length. Typically, during normal operation $n_{d}$ is in the range $2 \times 10^{10}$ to $3 \times 10^{10}$. In this manner the wakefield has been measured for C-band [27-33], X-band [22,3437], and $\mathrm{K}_{u}$-band [38] accelerating structures.

The two main approaches to wakefield suppression are now discussed in the following sections: heavy damping and moderate damping together with strong detuning in the cell frequencies.

\section{B. Heavily damped, low- $Q$ accelerating structures}

\section{Wakefield suppression at $X$-band}

In order to achieve $Q$ values of $\sim 10$ in the CLIC baseline design, waveguides are strongly coupled to the accelerator and damping material is located in close proximity to the accelerated beam. However, the $Q$ of the main accelerating mode is lowered by $\sim 10 \%$ by these waveguides which require large coupling to each accelerating cell. Furthermore, in order to ensure the electric and magnetic fields are not enhanced, all surfaces of the cell and waveguide are rounded. In particular, the region in which the waveguide enters the cell is convex. This ensures the magnetic field and the associated pulse surface heating temperature are kept within tolerance limits. A CLIC main linac cell provided with four damping waveguides is illustrated in Fig. 2. The wakefield of an accelerating structure constructed of 24 of these cells for the present baseline CLIC accelerating structure CLIC_G is illustrated in Fig. 3 [39,40]. Beam dynamics simulations performed with PLACET [41] indicate that the wakefield at the first trailing bunch and all subsequent bunches must be below $6 \mathrm{~V} /(\mathrm{pC} \mathrm{mm} \mathrm{m})$ for the present set of optimized CLIC parameters [19]. These bunches are spaced from their neighbors by $15 \mathrm{~cm}$ $(0.5 \mathrm{~ns})$. The results of simulations made with the finite difference code GDFIDL [42], displayed in Fig. 3, indicate that this requirement on the damping of the envelope of the wakefield has been met. ASSET data obtained from an earlier tapered damped structure (TDS) indicated good agreement between predicted values and those observed

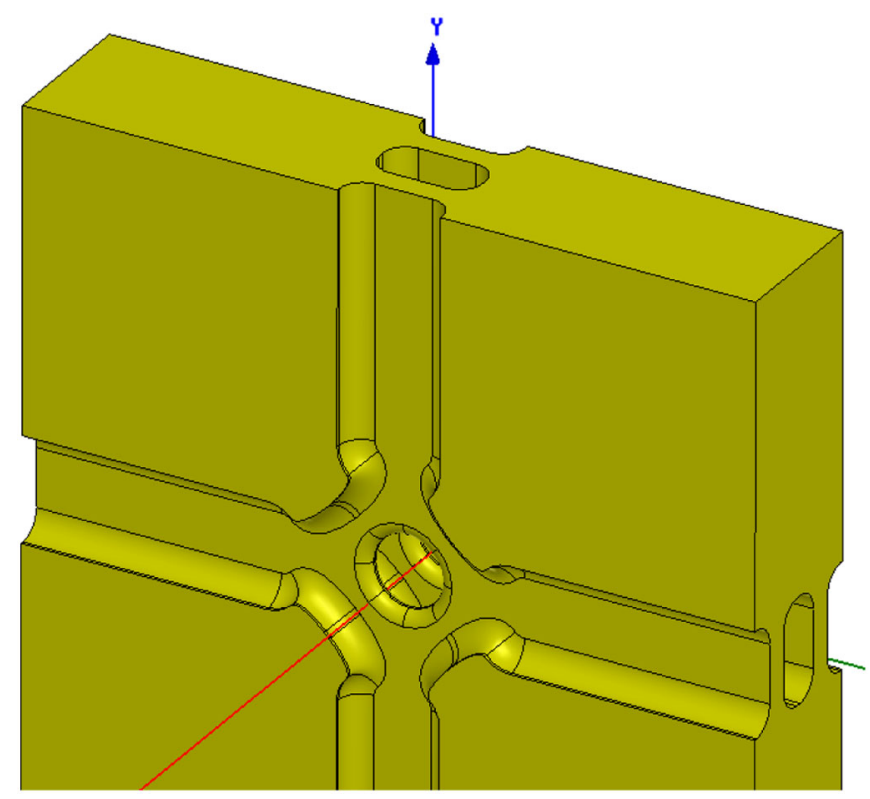

FIG. 2. (Color) CAD drawing of single cell for the main linac structures of CLIC $[39,40]$. 


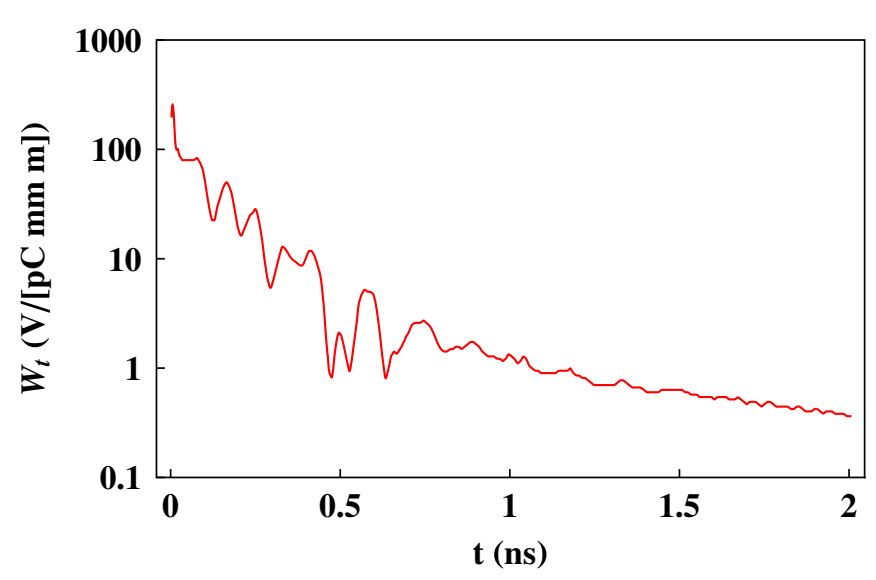

FIG. 3. (Color) Envelope of transverse wakefield for CLIC_G accelerating structure [39,40].

experimentally [38]. This 150-cell CLIC structure was provided with waveguide damping $(Q \sim 20)$ and operated at $15 \mathrm{GHz}$. There was, however, a mode a $\sim 7.6 \mathrm{GHz}$ which was not damped properly and this was attributed to a resonance external to the accelerating structure and was discovered after performing the measurement of the wake. Additional experimental tests on the present structure, which differs from TDS particularly in the shape of the outer walls, would provide further confidence in the efficacy of the damping. Furthermore, the pulse surface heating temperature rise is required to be less than $\sim 56^{\circ} \mathrm{K}[43-$ 45] and simulations indicate that the present CLIC_G $[39,40]$ structure is below these limits but it will require high power experimental studies in order to be acceptable for use in a linear collider. Reducing the magnetic field may be achieved with additional optimization or by utilizing an inherently different structure, such as that employed in the moderate damping scheme described in the next main section.

However, other means of achieving heavy damping are possible. In particular, radial slots in the irises of the cells can facilitate coupling out the dipole modes and this approach has been adopted for the CLIC drive beam accelerator (DBA) [46,47]. This approach is discussed in the next section.

\section{Wakefield suppression at S-band}

The CLIC accelerator relies on a two-beam acceleration concept [11,12]. One of which is the main accelerator, operating at a low current $(\sim 1 \mathrm{~A})$ and the other is a high current drive beam $(\sim 100 \mathrm{~A})$. The CLIC test facility CTF3, will demonstrate the power generation scheme and is equipped with DBA structures operating at an S-band frequency of $3 \mathrm{GHz}$. This frequency has been adopted as it capitalizes on the availability of $3 \mathrm{GHz}$ klystrons that have been liberated from LEP. These DBA structures for CTF3 must cope with high currents (3.5 A), long pulse lengths $(\sim 1.5 \mu \mathrm{s})$, and heavy beam loading $(\sim 98 \%)$. They have
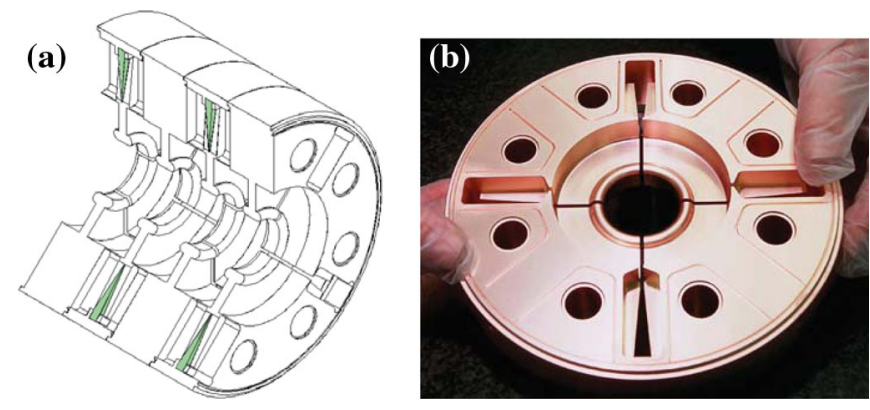

FIG. 4. (Color) Cross-sectional view of the slotted iris-constant aperture (SICA) geometry used for DBA wakefield damping (left) [46] and a prototype single cell (right) [47].

been designed to operate at a $2 \pi / 3$ phase advance per cell, at a moderate accelerating gradient of $\sim 6.5 \mathrm{MV} / \mathrm{m}$, and will consist of 32 main cells together with an additional two coupler cells. The CTF3 DBA consists of 16 of these slotted iris-constant aperture (SICA) accelerators, each of which is $1.22 \mathrm{~m}$ long. Illustrated schematically in Fig. 4(a) is a section of the SICA structure, together with a single fabricated cell in Fig. 4(b). The radius of the iris of each cell of the accelerating structure is maintained at a fixed value throughout, and these relatively large irises $(\sim 17 \mathrm{~mm})$ allow the short range wakefield to be minimized. The tip of each iris has the shape of a nose cone [48]. Detuning of the dipole modes and variation of the group velocity is achieved by varying the thickness of these nose cones. Radial slots emanating from the edge of the irises are terminated in ridged waveguide where tapered silicon carbide $(\mathrm{SiC})$ loads are located These slots function as waveguides which enable the wakefields to be efficiently coupled out of the structure, while ensuring the $Q$ of the fundamental mode suffers little perturbation. In this manner the $Q$ s of the dipole modes have been limited to $<20$. These slots enhance the electric field on the irises. However, this enhancement has been limited to a factor of $\sim 1.4$ [46] by rounding the edges using numerically controlled (NC) milling machines for this region. Also, "slot modes," at approximately $2 / 3$ the fundamental mode frequency can propagate, but these are more than adequately damped down to $Q$ s of $\sim 10$, and the kick factors have been found to be very small ( $\sim 5 \%$ of the lowest dipole kick factor) compared to those of the main structure. In addition, as the modes are strongly damped, coaxial pickups located within them can sample a portion of the power, and provide information on the position of the beam within the structure. This has been successfully demonstrated on a SICA structure consisting of four damping cells and two coupler cells [49]. A dipole mode at $4.25 \mathrm{GHz}$ was used in this manner and the beam position was determined to within an rms of $\sim 6 \mu \mathrm{m}$.

Another means of damping the wakefield, which relies on radially coupling out the higher order modes, is that of the choke mode method. This damping scheme, designed to operate at C-band, is described in the next subsection. 


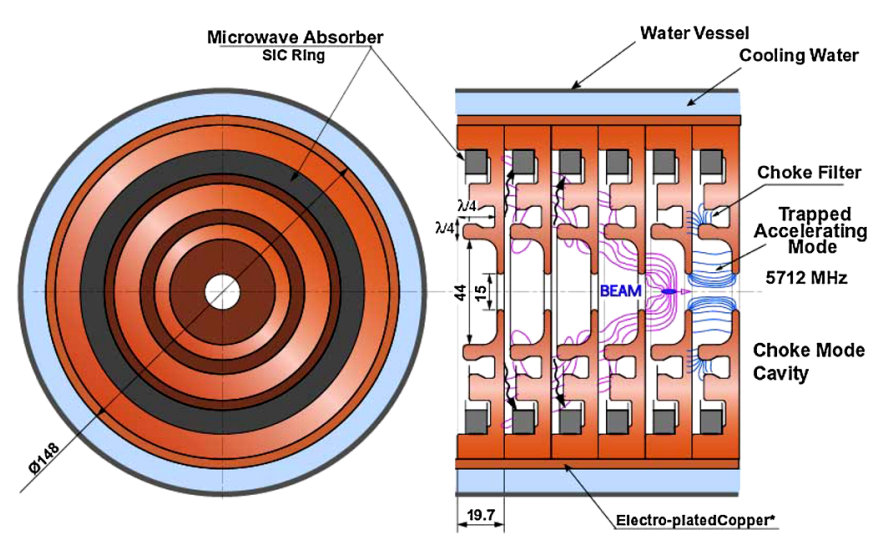

FIG. 5. (Color) C-band choke mode damped cavity [27-33].

\section{Wakefield suppression at C-band}

The choke mode [27-33] damping scheme, operating with a $3 \pi / 4$ phase advance per cell, has shown particular promise in damping transverse wakefields in C-band accelerating structures and is successfully operating with accelerating gradients of $37 \mathrm{MV} / \mathrm{m}$ at Spring-8 [50] in Japan. A $\mathrm{CAD}$ drawing illustrating the main features of the choke mode accelerator is illustrated in Fig. 5. This cavity allows the higher order modes to flow out through the slots and to be subsequently damped. The slots, transverse to the beam axis, function as radial waveguides and are designed to be a quarter wavelength long $(\lambda / 4)$ in order to reflect the e.m. field of the accelerating mode. Modes at an odd multiple of the accelerating frequency can also be trapped within the main accelerator cavity, including those in the vicinity of the modes which provide a strong transverse kick to the beam. Modifying the geometry of the slots shifts these parasitic modes to higher frequencies [29]. However, the prospects for operating this particular cavity at high gradients are not clear as high gradient studies have not been featured in the development of these cavities. Nonetheless, the dipole wakefield has been shown to be well damped in these structures from experiments performed at the accelerator structures facility (ASSET [33]) at the SLAC National Accelerator Laboratory.

Another strategy entails utilizing a structure which only moderately damps the $Q$ factors of the transverse modes but strongly detunes the modes of the cells in order to force the wakefield to decay in a prescribed manner. This approach is described in the next section.

\section{Resonant and nonresonant wakefield suppression with moderate damping}

The wakefield excited by a bunch train can be effectively canceled by arranging the frequencies of the modes excited in the cavities to either resonantly, or nonresonantly, suppress the wakefield.

The wakefield excites a series of modes and the overall wakefield can be represented as a sum over these modes, which have a sinusoidal dependence [14]. One means of resonant suppression entails ensuring the bunches in the train are located at the position of the zeros in the wakefield. The method is known as the zero-point crossing approach to minimizing the effect of the wakefield on beam dynamics. A further method entails ensuring the difference in two dipole frequencies excited are an integer multiple of the bunch repetition frequency. Both of these methods resonantly suppress the wakefield and will be sensitive to manufacturing errors, introduced inevitably in fabricating several thousand of these accelerating structures. In order to obtain some confidence that the wakefield impacts the emittance [51] and energy of the beam in a well-contained and limited manner, a careful study of the beam dynamics is required, with expected experimental errors included in the simulations.

\section{Wakefield suppression at S-band}

On the other hand, nonresonant wakefield suppression entails detuning the dipole frequencies excited by the beam and this is the method that has been extensively investigated in the context of the X-band NLC/GLC structures, for the S-band linear collider (SBLC) [52-55] and for the S-band Stanford linear accelerator (SLAC) [56]. The advantage of nonresonant wakefield over resonant suppression is that it does not freeze the bunch spacing to a particular value. In essence, nonresonant suppression forces the wakefield to decay for all bunch locations. This method of wakefield suppression can be understood by considering the momentum kick imparted transversely to the acceleration beam axis and this is governed by the transverse kick factor [14]. In forcing the wakefield to decay with a prescribed functional dependence, we begin by prescribing a specific dependence to the kick factor weighted density field with respect to frequency, $K d n / d f$. The inverse Fourier transform of $K d n / d f$ gives the wakefield experienced by the initial series of bunches in the train.

The linacs developed for SLAC in the 1960s were intended to accelerate electrons up to an energy of $40 \mathrm{GeV}$ through 960 constant gradient accelerator structures (each of which is $\sim 3 \mathrm{~m}$ long and consists of 86 cells) at a peak current of $100 \mathrm{~mA}$ and operated at a frequency of $2.856 \mathrm{GHz}$. During initial operation in the late 1960s, a BBU instability [17] was observed due to transverse deflecting modes being excited at $\sim 4.14 \mathrm{GHz}$. These dipole modes limited the operating current to a threshold of no more than $20 \mathrm{~mA}$. The focusing quadrupole magnets were subsequently redistributed and the cells towards the beginning section of a limited set of accelerator structures within sectors 1 and 2 (each of which is $333 \mathrm{ft}$ long) of the linac were detuned by squeezing them with a dimpling tool [57]. This enabled the threshold current to be raised to $\sim 60 \mathrm{~mA}$.

In the quest for higher energies, SBLC was designed to operate at an S-band frequency of $2.998 \mathrm{GHz}$ (a wavelength of $10 \mathrm{~cm}$ ) with 5032 structures, each of which was 
$6 \mathrm{~m}$ in length, and aimed at a center of mass energy at collision of $500 \mathrm{GeV}$. In the design of the SBLC, valuable experience gained on the SLAC linac motivated a study on the means to prevent BBU and emittance dilution occurring. Gaussian detuning of the 180-cell accelerating structures was investigated as a means of enforcing wakefield suppression. However, the final design opted for constant gradient structures operating at a loaded accelerating gradient of $17 \mathrm{MV} / \mathrm{m}$ (in which the modes will consequently be detuned with constant mode spacing) in which Gaussian detuning was not utilized. Over the length of the linacs, each of which consisted of 2516 accelerating structures, ten different structure types were envisaged, each one having a different frequency shift in the dipole modes. In this manner section-to-section detuning of constant gradient structures ensures coherent transverse momentum kicks were minimized and hence the influence on emittance dilution is reduced. This method was adopted in preference to enforcing an explicit Gaussian detuning distribution as it was simpler to implement. In addition, the tips of the irises of each of the cells were coated with Kanthal, a lossy material which ensured the dipole mode $Q$ was kept below 4000 and prevented a recoherence of the wakefield. A thin layer of 10 to $20 \mu \mathrm{m}$ was sufficient to achieve this damping and at the same time the shunt impedance of the accelerating mode was degraded by no more than 5\%. Two sets of higher order mode (HOM) couplers, each provided with four orthogonal waveguides, were used to monitor the radiation and from these signals structure alignment could be remotely measured. Each pair of facing waveguides facilitates the monitoring of one particular polarization. However, the SBLC work, largely centered at the DESY laboratory, was subsequently abandoned and all research efforts at that laboratory were refocused on a design which utilized superconducting cavities, known as TESLA [5]; this technology was later chosen for the ILC [4].

\section{Wakefield suppression at $X$-band}

For the NLC/GLC, several distributions were investigated [58], but a Gaussian distribution received a considerable amount of study. As the inverse Fourier transform of a Gaussian is also a Gaussian distribution, the wakefield decays rapidly according to $\sigma$, the prescribed standard deviation of the distribution. However, as each accelerating structure consists of a finite number of cells with a prescribed minimum and maximum iris radius, a sampling interval and a finite bandwidth are imposed upon the Gaussian distribution. In practice, the modes in a given accelerating cavity have a finite frequency separation. This means the wakefield will eventually recohere, according to the minimal separation of these modes. Thus, in practice detuning the modes of a cavity is not expected to be entirely sufficient to ensure adequate wakefield damping over the complete length of the bunch train. To overcome this inadequacy, and hence prevent the characteristic resurgence in the wakefield, moderate damping is employed. One possible means of affecting this damping is achieved by adding lossy material on each iris to bring down the copper $Q$ of the cavity. Another approach is to couple out a fraction of the dipole radiation through slots from each cell to manifolds attached to the walls of the accelerating structure. The latter was the approach followed by the NLC/GLC. Also, from monitoring the radiation in the manifolds, both the position of the beam can be ascertained and the alignment of cells can be remotely constructed [59-62]. The damping materials can be located remotely from the cells of the accelerator and will not be subject to high fields present when breakdown occurs in the cavity. In addition, the pulse surface heating temperature rise in the vicinity of the coupling slots is in general smaller than that in the heavily damped counterpart [63]. A CAD drawing of one of the several accelerating structures that was constructed to test the wakefield suppression properties is illustrated in Fig. 6.

This particular structure had an accelerating mode phase advance per cell of $5 \pi / 6$ and was part of a pair of structures that were measured simultaneously at the ASSET [37] facility at the SLAC National Accelerator Laboratory.

The wakefield excited by a multibunch particle beam can be suppressed by ensuring the modes excited in each of the cells of the structure differ in a prescribed manner. There is indeed considerable experience in this method, obtained through more than one and a half decades of research in the area during a collaborative program for the NLC/GLC involving the SLAC National Accelerator Laboratory, KEK National Laboratory, FNAL, and LLNL. The mechanism by which the wakefield, excited in multicell accelerator structures, couples to the attached manifolds can be understood by considering the characteristic dispersion curves of the cells. A Brillouin diagram for three

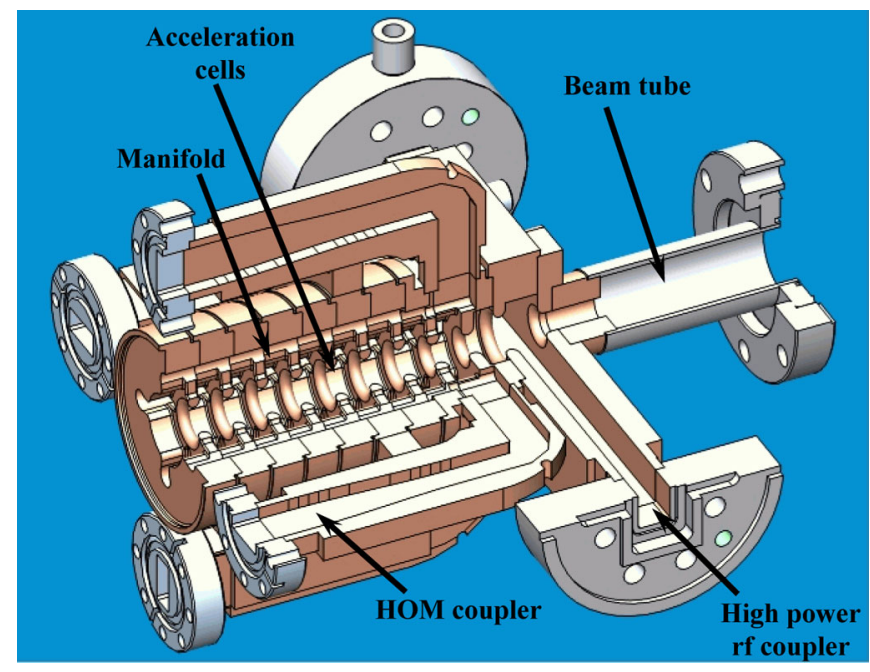

FIG. 6. (Color) CAD drawing illustrating a cross-sectional view of the output region of H60VG4SL17A. 


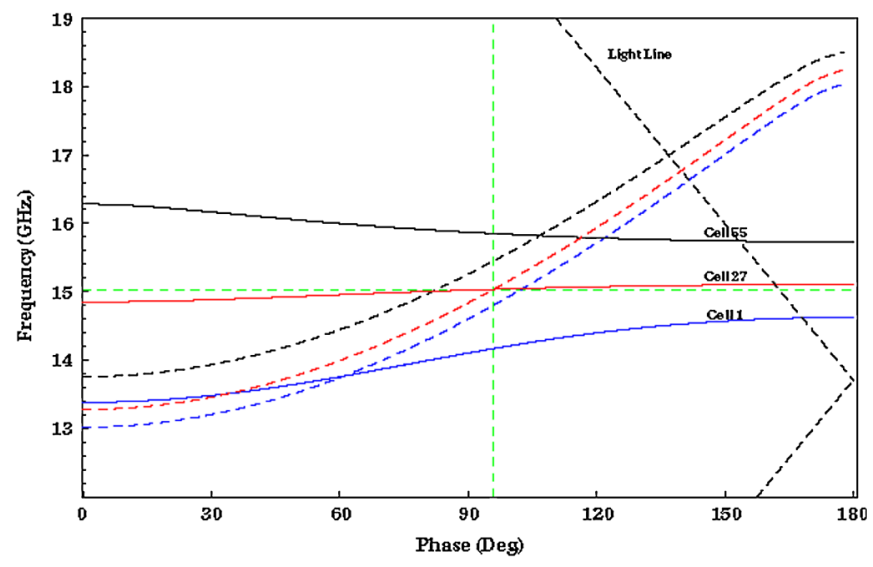

FIG. 7. (Color) Brillouin diagram for the high phase advance accelerator H60VG3.

representative cells out of a 55 cell structure, which operates at a fundamental mode accelerating phase advance of $5 \pi / 6$, known as H60VG3, is illustrated in Fig. 7. Here the light line is indicated by the black dashed line and the solid curves represent the dispersion curves for first, middle, and last cells prior to coupling to the attached manifolds. The manifold propagates TE waveguide modes which couple to the accelerator via slots in the walls of the cells. Manifold modes are indicated by the dashed curves. The introduction of coupling slots will force a manifold mode and cell mode to be coupled. However, in order to understand the manner in which the beam excites modes and couples them to the manifold, the uncoupled curves will be used. Strong coupling of the accelerator modes to the manifold modes occurs where the curves cross. For example, in the center of the accelerator, at cell 27, the manifold and dipole modes cross at the point indicated by the intersection of the horizontal and vertical green dashed lines (at a frequency of $15.3 \mathrm{GHz}$ and at corresponding phase advance of 95.9 degrees). Interpolating between the dipole curves shown at $0^{\circ}$ and $180^{\circ}$ indicates that the mode is localized to cells 20 to 34 (outside this domain the modes are in the characteristic "stopband" region). Also, the horizontal green dashed line intersects the light line at the synchronous point corresponding to cell 20 . Thus, the ultrarelativistic beam traversing the accelerator excites a dipole mode at cell 20 and couples out to the attached manifold, not in the immediate vicinity of the excitation, but further downstream, at the slot located in cell 27. From similar considerations, the coupling to all cells can be understood. The point at which the manifold and dipole modes intersect for each cell is illustrated in Fig. 8, together with the intersection of the light line with the dipole modes, and the location of the $0^{\circ}$ and $180^{\circ}$ phase advance for each cell. From these curves the mechanism of excitation and coupling can be understood for any of the cells. In general, the dipole mode in a damped and detuned accelerator is excited at a particular cell, is localized (or trapped) to within a

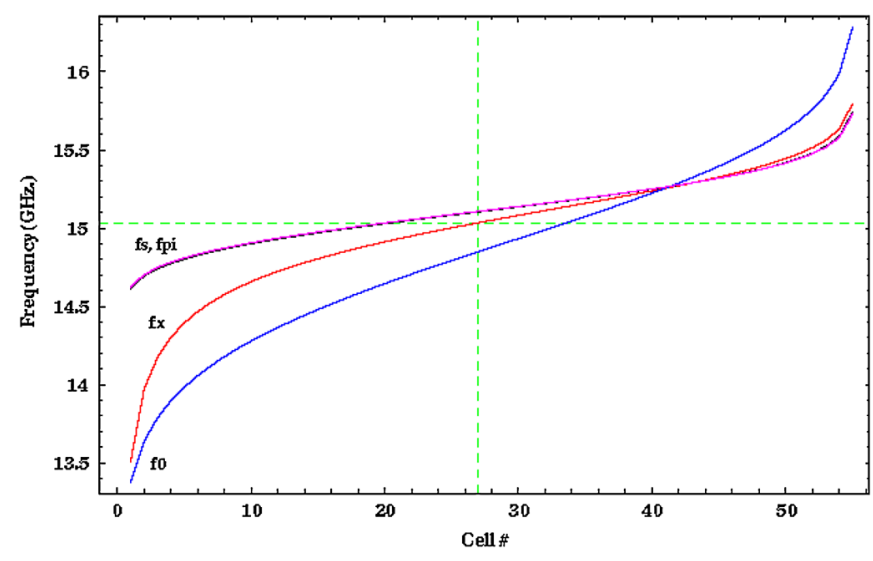

FIG. 8. (Color) Mode coupling parameters versus cell number for H60VG3. Here $f s$ indicates the location of the intersection of the light line with the cell mode and $f x$ the intersection of the manifold and cell mode. Also, $f 0$ and $f p i$ indicate the location of the $0^{\circ}$ and $180^{\circ}$ point in the cell modes.

limited number of cells, and it subsequently couples out to the attached manifold further down the accelerator.

The wakefield in these damped and detuned accelerating structures can be calculated by various means. In particular, finite element [64] and finite difference [42] computer codes can be used as tools to simulate the wakefield excited. However, these codes require substantial resources, in terms of memory and CPU time. In addition, they do not readily lend themselves to rapidly assessing the effect of changes in cell geometry which occur during the course of designing and testing these accelerating structures. Furthermore, during the period the initial structures were being fabricated, no computational tools of this form were available to model the complete accelerating structures. For this reason a circuit model [35,37] was developed which focused on the dominant first band dipole mode excited in the structures. This was subsequently supplemented with a spectral analysis $[37,65]$ of the wakefield.

At short time scales, an approximate method yields reasonably accurate results and this allows a rapid design of wakefield damping for the first few bunches. For short time scales, the damping due to the $Q$ resulting from coupling to the manifold can safely be ignored. This is clearly an excellent approximation for the medium damped structures used in the NLC/GLC program (where $Q \sim$ 500-1000) and for those envisaged for the alternate CLIC design. In this region, detuning alone accounts for the decay in the envelope of the wakefield.

The initial work in this area entailed detuning each of the modes in a 206 cell structure, such that the iris radius followed an erf (error function) distribution with cell number. In this manner the iris radius decreases smoothly down the accelerator. The resulting wakefield will initially decay with an approximately Gaussian distribution. However, this is not expected to be an ideal representation of a Gaussian for several reasons. In particular, the end points 
of the Gaussian, defined by the end cells in the structure, will give rise to a truncation in the Gaussian field. Furthermore, as there are a finite number of cells which sample the field then the modes which constitute the wakefield will eventually recohere. This recoherence in the modes will result in a resurgence in the wakefield. A measurement on a damped and detuned structure (DDS1), the first in a series provided with detuning of the cell frequencies and manifold damping, is illustrated in Fig. 9 along with theoretical predictions based on the circuit model and a spectral analysis of the wakefield. The cells were designed such that the mode density field $d n / d f$, rather than $K d n / d f$, was prescribed to be Gaussian. In this case, the initial decay is indeed Gaussian. However, finite sampling gives rise to a resurgence in the wakefield and this is evident for both DDS1 and for the detuned (DT) version of the accelerator in the region of $\sim 11 \mathrm{~m}$. The manifold damped structure, DDS1, also suffers from an additional feature which dilutes wakefield suppression further due to reflections which occur at the microwave ceramic window to the HOM loads. These windows had a reflection coefficient $S_{11} \approx 0.1$. Even though this is equivalent to a very small amount of power being reflected ( $99 \%$ transmitted), the wakefield is nonetheless sensitive to $S_{11}$ and hence the suppression in the wakefield is compromised. Simulations indicate that well-matched HOMs would enable the wakefield $5 \mathrm{~m}$ behind the first bunch to be at a level of $\sim 0.2 \mathrm{~V} /(\mathrm{pC} \mathrm{mm} \mathrm{m})$, rather than the predicted and observed level of $\sim 1 \mathrm{~V} /(\mathrm{pC} \mathrm{mm})$. For the NLC/GLC, beam dynamics simulations indicated that the wakefield must be below $1 \mathrm{~V} /(\mathrm{pC} \mathrm{mm} \mathrm{m})$ at the location of each bunch in the train, in order that the emittance dilution be kept below $5 \%$. Thus, DDS1 has met these limits, but manufacturing more than sixteen thousand, as required for a linear collider

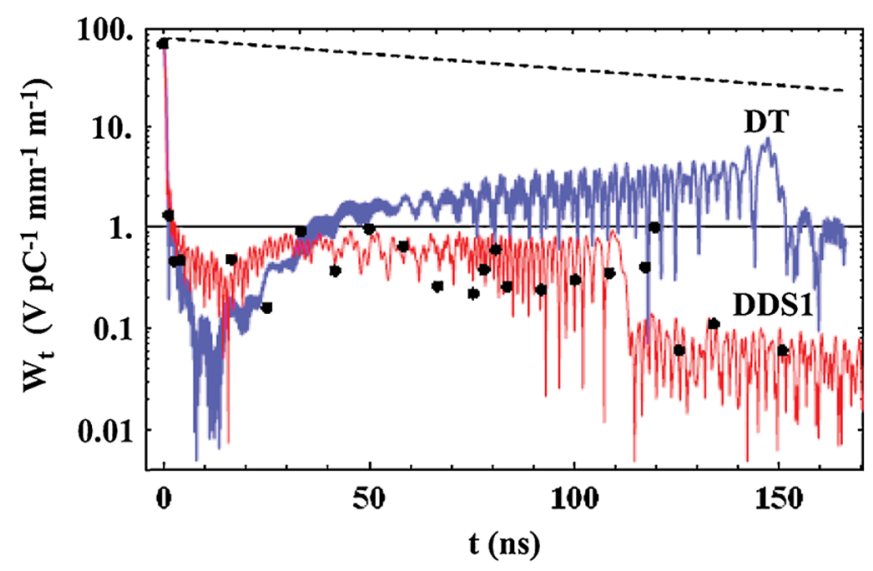

FIG. 9. (Color) Simulation of $W_{t}$, envelope of wakefield, for DDS1 (in red), the first damped and detuned structure, compared to a detuned structure DT (in blue). Also indicated are points obtained from an experimental measurement of the wakefield and the damping (indicated by the black dashed line) which is attributable to purely Ohmic losses. with a center of mass energy of $500 \mathrm{GeV}$, may introduce frequency errors which could cause the wakefield to fall short of this criterion.

The next major set of structures, DDS3 and DDS4, dispensed with these windows in order to improve overall suppression of the wakefield. Indeed, all subsequent structures were also designed without windows in the HOM load region. Furthermore, the kick factor [14] weighted density field $K d n / d f$, rather the density field, was prescribed to be a Gaussian field. This enables the spectral field (the inverse transform of the wakefield) to be symmetrical and the minima in the wakefield to be well defined. The bandwidth and standard deviation of the Gaussian was also modified with a view to optimizing the decay in the wakefield. However, in manufacturing each of the 206 cells, which composed DDS3 and DDS4, a significant random error was introduced during the machining process. This impacted the overall wakefield suppression and prevented an observation of the well-defined minima in the wakefield. Even though it is far from the design ideal, it still is below $1 \mathrm{~V} /(\mathrm{pCmmm})$ and it is illustrated in Fig. 10. These errors obtained during the fabrication of the cells were not well defined and so errors were introduced at a nominal level into the spectral field model of the structure and increased until the wakefield of the model matches the measured values. This led to ascribing an rms error of $12 \mathrm{MHz}$ to the cell frequencies. However, as the minimum mode spacing is $\sim 8 \mathrm{MHz}$, the wakefield is adversely affected due to these large random errors introduced during the manufacturing process. The minimum mode spacing effectively sets the maximum allowable frequency errors. Furthermore, the monopole accelerating mode has stringent requirements on acceptable frequency errors which are imposed in order to obtain an acceptable efficiency in accelerating the charged particle beam. For the NLC/GLC this imposed an rms frequency error of no more than $\sim 1 \mathrm{MHz}$ on the monopole

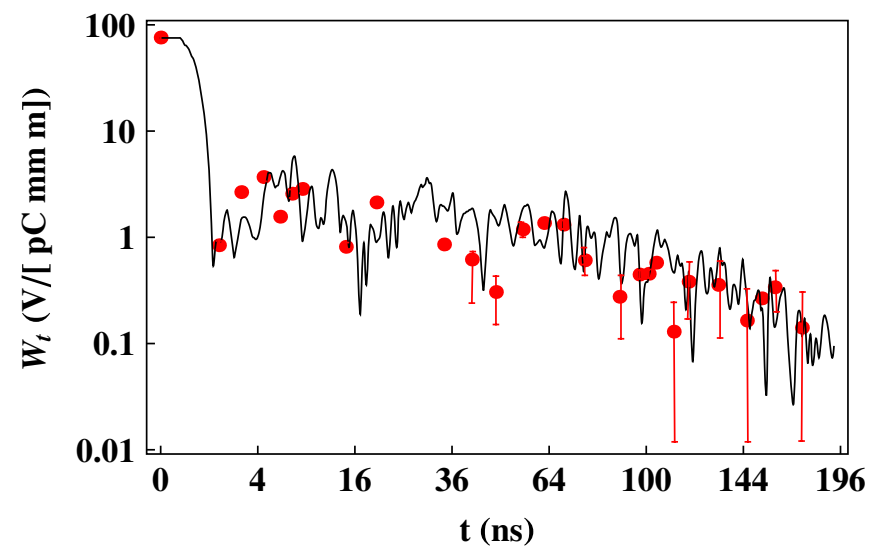

FIG. 10. (Color) Spectral simulation (solid line) and experimental measurements (red dots) of the envelope of the wakefield for DDS3. Random errors in the cell frequencies are also included in the simulation with an rms value of $12 \mathrm{MHz}$. 
mode. Once this limit is imposed on the manufacturing process, similar frequency bounds will follow for the dipole mode.

The next series of structure focused on improving the shunt impedance and $Q$ factor of the accelerating mode. This was achieved by carefully shaping the iris and cavity of each cell of the accelerating structure. In this manner, the shunt impedance was improved by $\sim 10 \%$. This 206 cell accelerator was known as RDDS1. However, in fabricating these structure systematic errors, rather than random errors, were inadvertently introduced into a limited number of cells in the structure. These errors occurred during the diffusion bonding process in which the cells are bonded by heating them to a temperature little over $1050^{\circ} \mathrm{C}$. During this process the structure is supported with a series of stainless steel collars. These collars had a different expansion coefficient from the copper structure and hence they caused a larger expansion of the cells in this region. This expansion was confirmed, prior to measuring the wakefield in ASSET [22], by using a coordinate measurement machine to record the outer radius; the resulting inner radius deviation was inferred from that of the outer radius. This gave a predicted shifting in the synchronous frequencies of the middle cells of approximately $30 \mathrm{MHz}$. This was subsequently confirmed with a "bead-pull" phase measurement of the structure [66]. This systematic shift in the cell frequencies is particularly damaging as it is located in the center of the structure, the region of minimum mode spacing, which is $\sim 7 \mathrm{MHz}$. The mode spacing together with the kick factor weighted density of modes, $K d n / d f$, are illustrated in Fig. 11. Frequency shifts of a similar magnitude also occurred in the end cells. However, as the mode spacing towards the ends of the distribution is more than $100 \mathrm{MHz}$, the frequency errors had little impact on the wakefield. The resulting experimental measurement of the wakefield, together with that of the predicted wake for a detuned structure (DT) and a rounded damped de-

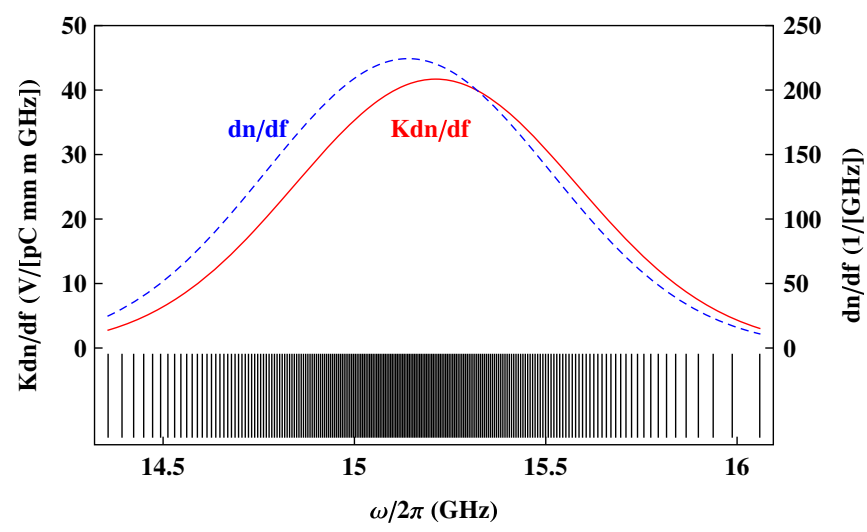

FIG. 11. (Color) Smoothed undamped kick factor weighted density function $K d n / d f$ (in red), mode density function $d n / d f$ (in blue and dashed), and the location of individual modes (indicated by vertical black lines).

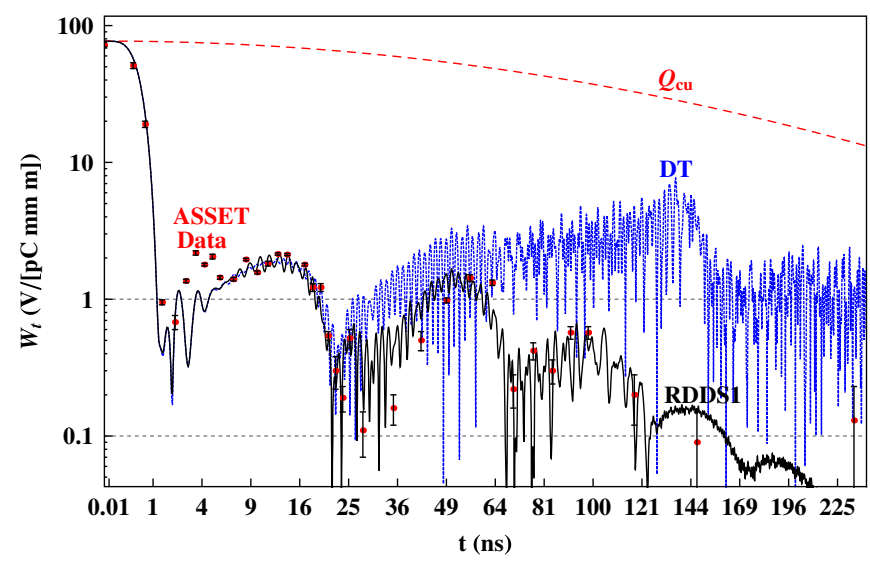

FIG. 12. (Color) Envelope of transverse wakefield for RDDS1 due to frequency errors obtained in fabricating a complete 206 cell structure [22]. The predicted wakefield is indicated by the solid black line and the red dots correspond to experimentally determined values. The design goal of $1 \mathrm{~V} /(\mathrm{pC} \mathrm{mm} \mathrm{m})$ and the limit in the accuracy of the measurement of $0.1 \mathrm{~V} /(\mathrm{pC} \mathrm{mm} \mathrm{m})$ are indicated by horizontal dashed lines.

tuned structure (RDDS1) is illustrated in Fig. 12. The circuit model provides a good prediction of the wakefield as the general properties of the wakefield are reproduced. The horizontal lines at $1 \mathrm{~V} /(\mathrm{pCmmm})$ and $0.1 \mathrm{~V} /(\mathrm{pC} \mathrm{mmm})$ represent the level at which the wakefield must be damped and the measurement accuracy of the ASSET measurement setup, respectively. Clearly the wakefield rises above $1 \mathrm{~V} /(\mathrm{pCmm} \mathrm{m})$ and this is due to the frequency shift in the central cells. Replacing the supports with materials of similar expansion coefficients to that of copper will remove this frequency shift and this was incorporated in the next series of structures.

The next series of structures were designed with a view to achieving high gradient operation, while minimizing electrical breakdown, and were known as the high phase advance or HDDS series. Inspection of the (R)DDS series indicated that severe damage due to electrical breakdown had been occurring in a number of cells and this motivated the HDDS accelerators. The initial design was prompted from a realization that the worst damage occurred in the cells with high group velocity and hence the group velocity of subsequent structures was reduced by moving from a $2 \pi / 3$ phase advance per cell to a $5 \pi / 6$ phase advance. Furthermore, the input power was reduced by limiting the number cells per accelerating structure to 55, rather than the 206 used in all previous structures. In addition, the ratio of surface field to accelerating monopole field was carefully optimized to be $\sim 2$ over the complete accelerating structure by modifying the cell geometry. This limits the high electric field region along the surface of the cavity. Other issues affecting electrical breakdown include surface processing, and gradually bringing the accelerating field up to the required value over a period of time by appropriate high field processing. A detailed discussion of these issues 


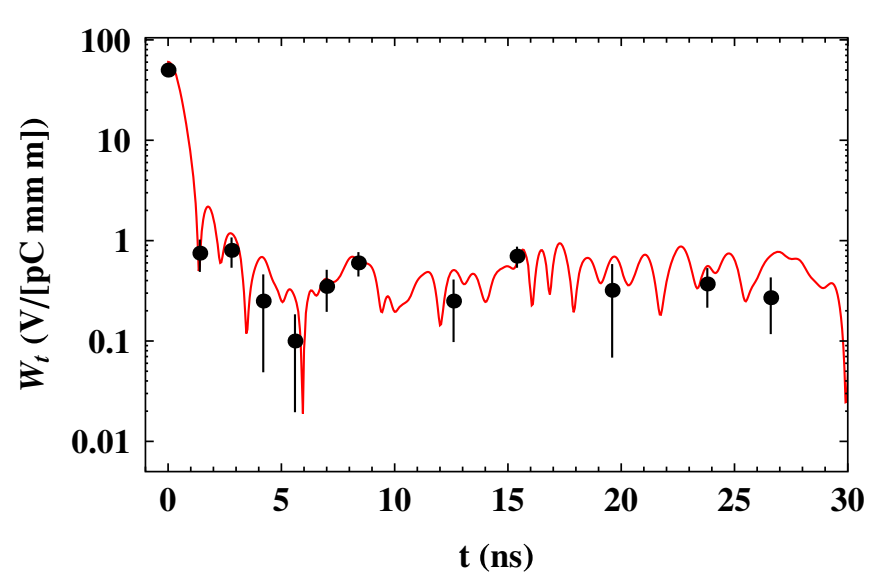

FIG. 13. (Color) Envelope of wakefield for H60VG4SL17A/B [37]. The wake predicted with the circuit model is indicated with the solid red line and the experimentally determined values by points.

is beyond the scope of this paper. However, breakdown issues in the context of high gradient accelerator cavities are a topic of ongoing research and we refer the reader to [67].

The wakefield in reduced-length accelerating structures, with a concomitant reduction in surface field, were known as H60VG4SL17A/B. These structures were $60 \mathrm{~cm}$ in length and consisted of 55 cells. However, as these structures had an almost fourfold reduction in the number of cells compared to DDS1, the sampling of the uncoupled kick factor weighted density field, $K d n / d f$, is expected to be severely limited. Indeed, this limited sampling dilutes the effectiveness of the wakefield suppression significantly. For this reason, modes from successive accelerating structures were interleaved. Fourfold interleaving allowed excellent wakefield suppression to be achieved as this allowed the $K d n / d f$ field to sample mode than 220 cells. In order to verify the efficacy of the design, a twofold interleaved design was performed. This design initial was made by choosing the uncoupled cell frequencies, according to the coupled mode frequencies. As discussed earlier, the coupled cell frequencies determine the long-term behavior of the wakefield and the uncoupled ones the short term behavior over the region of the first few bunches in the train. The two structures, tested simultaneously at ASSET, were part of a nonfully optimized design which relied on the frequencies from the uncoupled optimization. The results from both the prediction, based on the circuit field analysis and experimental values, are displayed in Fig. 13. It is clear that the circuit model provides a good description of the wakefield excited by the beam. Furthermore, this wakefield, even though nonoptimum, satisfies the beam dynamics criterion which, for the NLC/GLC, prescribed that the wakefield must be below $1 \mathrm{~V} /(\mathrm{pC} \mathrm{mm} \mathrm{m})$ at the location of each bunch. Noninterleaving does not allow adequate suppression, as in this case after several trailing bunches the wakefield rises above unity. This was the final accelerator structure, designed and tested as part of the NLC/GLC program, equipped with both manifold wakefield suppression and detuning in the cell frequencies. After this structure was tested, a decision was made by the International Technical Review Panel (ITRP) [68] to shift the focus from a dual effort on SC and NC research towards a single concerted approach based on SC technology with an anticipated center of mass energy of $0.5 \mathrm{TeV}$, at the point of collision, with a potential upgrade to $1 \mathrm{TeV}$.

However, in order to reach high energies, a realistic path to this goal is provided by higher gradient linacs. CLIC aims at a center of mass collision of $3 \mathrm{TeV}$ using an accelerating gradient of $100 \mathrm{MV} / \mathrm{m}$. The present baseline design for wakefield suppression in the CLIC linacs is to use heavy damping and this was described in the previous section. However, an alternate design based on moderate wakefield suppression $(Q \sim 500)$ together with strong detuning is being developed. Here the challenge is to achieve adequate wakefield suppression at the first and subsequent trailing bunches, with a more stringent interbunch spacing of $0.5 \mathrm{~ns}$ (rather than the $1.4 \mathrm{~ns}$ used in the NLC/GLC). A design, utilizing eightfold interleaving, with this damping in mind has resulted in a well-suppressed wakefield, and is illustrated in Fig. 14. However, this required a dipole bandwidth of $3 \mathrm{GHz}$ and gives rise to unacceptably large surface electric fields on the walls of the cavity together with a large group velocity in the monopole mode. Thus, even though this structure meets the beam dynamics requirements imposed on wakefield suppression, it does not fulfill the electrical breakdown criterion [43-45] which limits the electric and magnetic surface fields. In the design of the present series of CLIC accelerating structures, both the surface electric and magnetic fields are minimized by suitably choosing the shape of the cells. This has resulted in CLIC_G having a surface electric field to accelerating field ratio of approximately two. The Poynting vector corresponding to the complex power flow must also be minimized $[69,70]$. These electrical design constraints limit the electrical breakdown to acceptable levels and ensures the pulse surface heating temperature rise on the surface of the cavity is kept to a prescribed level. The present design limits the pulse surface heating temperature rise to $56^{\circ} \mathrm{K}$ [43-45]. Based on these design constraints, an optimization of the wakefield is illustrated in Fig. 15. Here the number of cells has been reduced to more closely match the present CLIC design of 24 per structure. In order to limit the emittance dilution, it is proposed to vary the cell frequencies in a systematic manner from structure to structure with a view to ensuring a coherent growth in the emittance is prevented from occurring by locating the first few bunches in the region of zero crossing [71] in the wakefield. In this manner, the limited damping of the first few bunches may be overcome. A comprehensive series of beam dynamics simulations will address the question of whether the dimension tolerances required for the cells of each accelerating structure are realistic. 

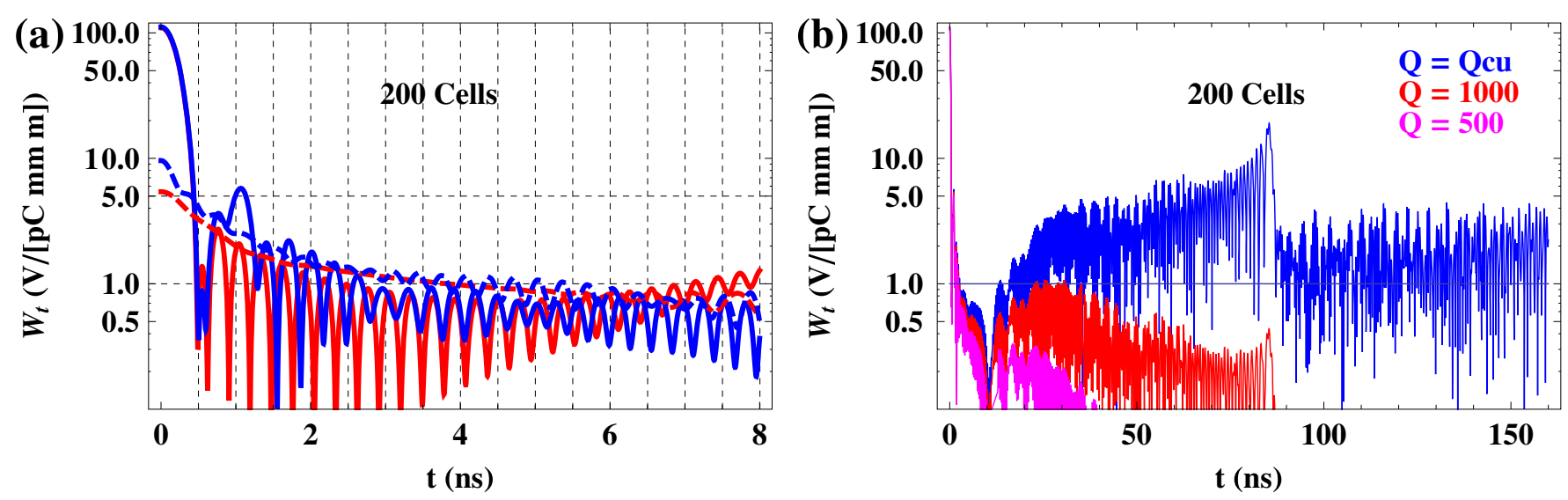

FIG. 14. (Color) Envelope of wakefield based on moderate damping and detuning for CLIC. Eightfold interleaving of a 25-cell structure results in 200 equivalent cells. The wakefield (excluding Ohmic losses) for the first 16 bunches is shown in (a) using an uncoupled model (in blue) and a coupled model (in red). Also shown in (b) is the wake for all subsequent bunches with various $Q$ values.
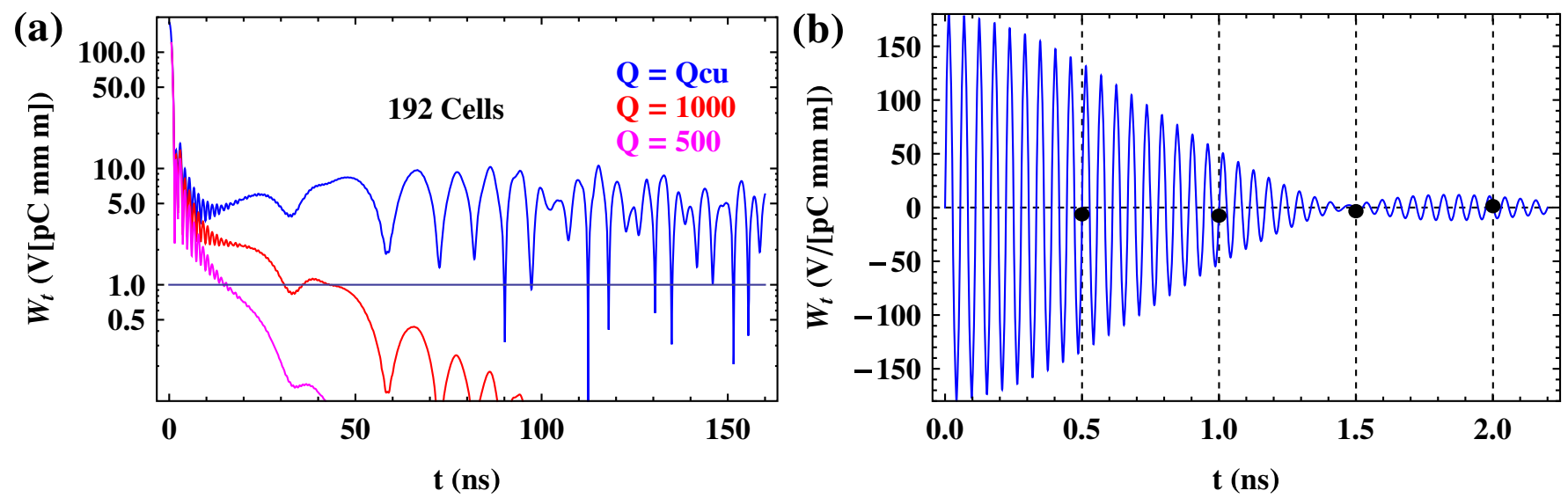

FIG. 15. (Color) Envelope of wakefield based on moderate damping and detuning for CLIC. Eightfold interleaving of a 24-cell structure results in 196 equivalent cells. The envelope of the wakefield for various $Q$ values is shown in (a) and the amplitude of the wakefield is shown in (b), demonstrating the bunches are located at the zero-crossing points in the wake.

Beam dynamics simulations performed as part of the NLC/GLC program indicated the frequency tolerances imposed on the structures can be quite loose. A summary of these beam dynamics simulations is provided in the next section

\section{BEAM DYNAMICS}

In order to assess the influence of both random and systematic errors, the multibunch beam is injected into the linac with an initial offset of $\sigma_{y}$ and the progress of the beam is tracked down the complete linac. Various codes are available for this purpose. During the NLC/ GLC program LIAR [72] in particular was used intensively to simulate the overall beam dynamics properties of the machine subjected to a series of dimensional and alignment errors. Mapping the progress of the beam down the complete linac, consisting of more than 5000 accelerating structures per linac, allows the emittance dilution to be monitored and to ascertain whether or not the damping is sufficient. This procedure was employed for several of the accelerating structures. The results of this beam dynamics study for H60VG4SL17A/B, the last accelerating structure tested at ASSET for wakefield suppression, are illustrated in Fig. 16. The final emittance dilution, together with the rms of the sum wakefield, is illustrated for small changes in the bunch spacing. In all cases, random frequency errors are incorporated into a set of 50 accelerating structures and are randomly distributed along the entire linac $(\sim 10 \mathrm{~km}$ long). The beam was injected into the linac with an offset of approximately one $\sigma_{y}$, with an energy of $5 \mathrm{GeV}$ and the progress of the beam is monitored as it traverses the entire linac. This simulation includes a relatively large cell-tocell frequency error with an rms value of $20 \mathrm{MHz}$. This large frequency error allows a study of the impact of 


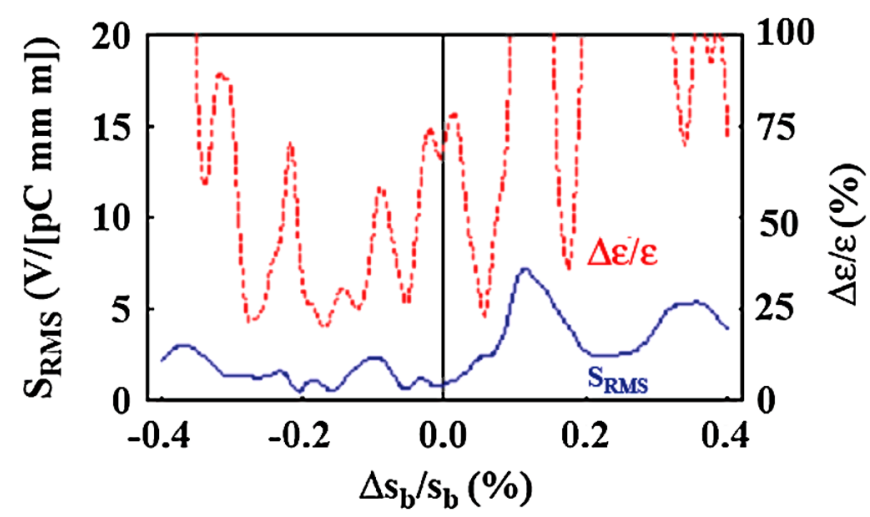

FIG. 16. (Color) Emittance dilution (illustrated by the red dashed curve) versus the percentage change in the bunch spacing. Also shown is the corresponding rms of the sum wakefield (by the solid blue curve).

relaxed manufacturing tolerances on the design of the linac. At the nominal NLC/GLC bunch spacing of $1.4 \mathrm{~ns}$ the emittance is diluted by approximately $70 \%$. This is reduced by a factor of 2 or more if the bunch spacing is changed by a small fractional amount. These small changes in the bunch spacing correspond to a shift in all the dipole frequencies of the accelerator structure. Thus, the emittance dilution for a $\pm 0.05 \%$ change in the bunch spacing can be achieved by shifting the dipole mode frequencies by $\sim 8 \mathrm{MHz}$. In practice, this is readily obtained from small changes in the geometrical parameters of the iris. A further means to minimize the emittance dilution is to introduce emittance bumps into the linac. Nonetheless, the twofold interleaved set of structures gives a final emittance dilution (with suitable shifting of the mode frequencies) of $\sim 25 \%$ which is close to the NLC emittance budgeted value of $10 \%$ for long-range wakes in the main X-band linacs.

Other codes, such as PLACET [41] and LUCRETIA [73] in particular, are now being used in beam dynamics simulations of the ILC and CLIC.

\section{FUTURE PROSPECTS AND CONCLUSIONS}

There are two main strategies of wakefield suppression for lepton collider applications. One entails heavy damping, which amounts to designing an accelerator structure which has a small $Q$ value $(\sim 10)$; choke-damped and waveguide damped structures have been designed for this application. The other method entails moderate damping, with $Q$ s ranging from 500 to 1000 , together with strong detuning in the cell frequencies. The latter, damped and detuned (DDS) method of wakefield suppression has the potential advantage that the loads can be remotely located from the accelerator cells. Also, in the quest for high gradient acceleration, the DDS approach offers the potential for a lower maximum pulse surface heating temperature rise. This approach is relatively mature, in that it has received more than one and a half decades of focused research from several international laboratories for the NLC/GLC program and this has resulted in a suite of structures which have been tested for high gradient applications and for wakefield suppression. It is interesting to note that beam dynamics considerations allow relatively loose random errors on frequency tolerances, which are as high as $20 \mathrm{MHz}$. On the other hand, the accelerating mode has a stringent tolerance of $1 \mathrm{MHz}$. Thus, provided the fundamental accelerating mode tolerance is met, the higher order mode wakefield tolerance will be relatively straightforward to achieve. However, systematic errors in the frequency tolerances are more stringent on the dipole mode and are sensitive to geometrical locations within the accelerating structure.

As far as high gradient applications are concerned, the baseline design for CLIC, operating at an accelerating gradient of $100 \mathrm{MV} / \mathrm{m}$, entails heavy damping but may have a significant pulse surface heating temperature rise in the vicinity of the coupling interface between the damping waveguide and the accelerating structure. The moderately damped DDS alternate method offers the potential of reduced pulse surface heating temperature rise and will allow a portion of the wakefield to be monitored from the HOM ports in order to remotely diagnose both the position of the beam and the cell-to-cell alignment. At this stage, both means of wakefield suppression should be pursued in order to thoroughly assess their applicability. Experimental testing, using realistic pulse lengths and at the high gradients planned for the linear collider, will be the final test on the suitability of these techniques.

\section{ACKNOWLEDGMENTS}

Thanks are due to international colleagues and collaborators, too numerous to mention individually, whom I have worked with over the years on the analysis of wakefields. I am grateful to Walter Wuensch for reviewing a draft of this paper and for providing a context to the current CLIC work. Significant parts of this work were supported by the U.S. Department of Energy under Contract No. DEAC02-76SF00515. The most recent parts of this research leading to these results have received funding from the European Commission under the FP7 Research Infrastructures Grant Agreement No. 227579.

[1] Lyndon Evans and Philip Bryant, JINST 3, S08001 (2008).

[2] Lyndon Evans, New J. Phys. 9, 335 (2007).

[3] International Linear Collider Technical Review Committee (ILC-TRC) Second Report No. SLAC-R606, 2003.

[4] International Linear Collider Technical Review Final Report, 2004, http://www.linearcollider.org.

[5] R. Brinkman et al., The TESLA Technical Design Report, DESY Report No. 2001-33, 2001. 
[6] R. L. Geng, Physica C (Amsterdam) 441, 145 (2006); 441, 145 (2006).

[7] T. Saeki, SMTF Collaboration Meeting, Fermilab, Batavia, IL, 2005.

[8] N. Phinney et al., Report No. SLAC-R-571, 2001.

[9] J. Wang and T. Higo, ICFA Beam Dynamics Newsletter 32, 27 (2003), http://icfa-usa.jlab.org/archive/newsletter/ icfa_bd_nl_32.pdf.

[10] H. Braun et al., CLIC-Note 764, 2008.

[11] Frank Tecker, J. Phys. Conf. Ser. 110, 112005 (2008).

[12] Jean-Pierre Delahaye, J. Phys. Conf. Ser. 110, 012009 (2008).

[13] G. Riddone (private communication).

[14] P. B. Wilson, SLAC Report No. SLAC-PUB-4547, 1987.

[15] P. B. Wilson, Rev. Accel. Sci. Technol. 1, 7 (2008).

[16] V. Balakin, S. Novakhatsky, and V. Smirnov, in Proceedings of the 12th International Conference on High Energy Accelerators, Fermilab, Batavia, IL, 1983 (Fermi National Laboratory, Batavia, IL, 1983), p. 119.

[17] K. Yokoya, DESY Report No. 86-084, 1989.

[18] H. Henke, CERN-LEP-RF/88-49, CLIC-Note-78, 1988.

[19] A. Grudiev, H. H. Braun, D. Schulte, and W. Wuensch, in Proceedings of the 24th Linear Accelerator Conference (LINAC08), Victoria, British Columbia, Canada, 2008.

[20] R. M. Jones et al., in Proceedings of the 21 st International Linac Conference, Gyeongju, Korea, 2002 (Pohang Accelerator Laboratory, Pohang, Korea, 2002).

[21] R. M. Jones, in Proceedings of the 22nd Linear Accelerator Conference (LINAC04), Lübeck, Germany, 2004 (Report No. SLAC-PUB 10682, 2004).

[22] R. M. Jones et al., New J. Phys. 11, 033013 (2009).

[23] M. Sands and J. Rees, SLAC Report No. PEP-95, 1974.

[24] F. Caspers, Bench Methods for Beam-Coupling Impedance Measurement, Lecture Notes in Beams: Intensity Limitations (Springer, Berlin, 1992), Vol. 400.

[25] N. Baboi et al., in Proceedings of the 8th European Particle Accelerator Conference, Paris, 2002 (EPS-IGA and CERN, Geneva, 2002).

[26] R.M. Jones et al., Proceedings of the 8th European Particle Accelerator Conference, Paris, 2002 (Ref. [25]).

[27] T. Shintake, Jpn. J. Appl. Phys. 31, L1567 (1992).

[28] T. Shintake, in Proceedings of the 17th Linear Accelerator Meeting, Sendai, Japan, 1992; KEK Report No. 92-66, 1992

[29] T. Shintake, in Proceedings of the Particle Accelerator Conference, Washington, DC, 1993 (IEEE, New York, 1993).

[30] T. Shintake, H. Matsumoto, and H. Hayano, in Proceedings of the 17th International Linear Accelerator Conference (LINAC94), Tsukuba, Japan, 1994 (KEK, Japan, 1994).

[31] H. Matsumoto, T. Shintake, N. Akaska, K. Watanabe, H.S. Lee, and O. Takeda, in Proceedings of the 5th European Particle Accelerator Conference (EPAC 96), Sitges, Barcelona, Spain, 1996, TUP069G, pp. 489-492.

[32] H. Matsumoto, T. Shintake, and N. Akasaka, in Proceedings of XIX International Linac Conference LINAC98, Chicago, Illinois, USA, 1998, MO4085.

[33] T. Shintake et al., in Proceedings of the 18th Particle Accelerator Conference, New York, 1999 (IEEE, New York, 1999).
[34] C. Adolphsen et al., Phys. Rev. Lett. 74, 2475 (1995).

[35] R. M. Jones et al., Proceedings of 5th European Particle Accelerator Conference (EPAC 96), Sitges, Spain, 1996 (Report No. SLAC-PUB 7187, 1996).

[36] C. Adolphsen et al., Report No. SLAC-PUB-8174, 1999.

[37] R. M. Jones et al., Phys. Rev. ST Accel. Beams 9, 102001 (2006).

[38] I. Wilson et al., in Proceedings of the European Particle Accelerator Conference, Vienna, 2000 (EPS, Geneva, 2000).

[39] W. Wuensch, in Proceedings of X-band Structures and Beam Dynamics Workshop (XB08), Cockcroft Institute, UK, 2008.

[40] W. Wuensch, in Proceedings of the 11th European Particle Accelerator Conference, Genoa, 2008 (EPS-AG, Genoa, Italy, 2008).

[41] E. D'Amico et al., in Proceedings of the Particle Accelerator Conference, Chicago, IL, 2001 (IEEE, New York, 2001).

[42] W. Bruns, in Proceedings of the Particle Accelerator Conference, Vancouver, BC, Canada, 1997 (IEEE, New York, 1997).

[43] The criterion of ensuring the pulse temperature rise is kept below $56^{\circ} \mathrm{K}$ is based on the semiempirical experimental and simulation (unpublished) work of S. Heikkinen, http:// clic-meeting.web.cern.ch/, CLIC Meeting, June 26th, 2003. Material characterization studies relating to pulse surface heating temperature rise as part of the CLIC study are G. Arnau-Izquierdo et al., Proceedings of Particle Accelerator Conference (PAC07), Geneva, Switzerland, 2007 (Report No. CLIC-Note-725, 2007); Fatigue 2007 Conference, Cambridge, UK, 2007 (Report No. CLICNote-708, 2007).

[44] A. Grudiev and W. Wuensch, Proceedings of the 24th Linear Accelerator Conference (LINAC08), Victoria, Canada, 2008 (Ref. [19]), THP062.

[45] A. Grudiev and W. Wuensch, Proceedings of the 22nd Linear Accelerator Conference (LINAC04), Lübeck, Germany, 2004.

[46] E. Jensen, Proceedings of the 21st International Linac Conference, Gyeongju, Korea, 2002 (Ref. [20]).

[47] E. Jensen, Proceedings of the Particle Accelerator Conference, Chicago, IL, 2001 (Ref. [41]).

[48] G. Carron, E. Jensen, M. Luong, A. Millich, E. Rugo, I. Syratchev, and L. Thorndahl, Proceedings of the 20th Linear Accelerator Conference (LINAC00), Monterey, CA, USA, 2000, TUA16, pp. 416-418.

[49] J. Prochnow, E. Jensen, and W. Wuensch, Proceedings of the 2003 Particle Accelerator Conference (PAC03), Portland, Oregon, 2003, WPPB025, pp. 2467-2469.

[50] T. Inagaki, Proceedings of the 24th Linear Accelerator Conference (LINAC08), Victoria, Canada, 2008 (Ref. [19]).

[51] A. Mosnier, Report No. DAPNIA-SEA-93-19, 1993.

[52] R. Brinkmann, Linear Collider Conceptual Design Report, Chapter 4: S-Band Linear Collider, 1997, http://www. desy.de/lc-cdr/.

[53] N. Holtkamp, in Proceedings of the Particle Accelerator Conference, Dallas, TX, 1995 (IEEE, New York, 1995).

[54] N. P. Sobenin et al., Proceedings of the 18th International Linear Accelerator Conference (LINAC 96), Geneva, 
Switzerland, 1996, THP66.

[55] P. Hulsmann et al., Proceedings of the 5th European Particle Accelerator Conference (EPAC 96), Sitges, Spain, 1996, TUP044L.

[56] The Stanford Two-Mile Accelerator, edited by R. B. Neal (W.A. Benjamin, Inc., New York, 1968).

[57] R. H. Helm et al., Proceedings of the Particle Conference, Washington, DC, 1969 (Report No. SLAC-PUB-563, 1969).

[58] R. M. Jones et al., in Proceedings of the 20th International Linac Conference (Linac 2000), Monterey, California, 2000 (Report No. SLAC-PUB-8609, 2000).

[59] M. Seidel et al., Nucl. Instrum. Methods Phys. Res., Sect. A 404, 231 (1998).

[60] R. M. Jones et al., in Proceedings of the 7th Workshop on Advanced Accelerator Concepts, Lake Tahoe, CA, USA, 1996 (Report No. SLAC-PUB 7388, 1996).

[61] R. M. Jones et al., Proceedings of the Particle Accelerator Conference, Vancouver, BC, Canada, 1997 (Ref. [42]).

[62] S. Döebert et al., in Proceedings of the 21st Particle Accelerator Conference, Knoxville, 2005 (IEEE, Piscataway, NJ, 2005).

[63] Z. Li (private communication).
[64] Z. Li et al., in Proceedings of the 9th European Particle Accelerator Conference, Lucerne, 2004 (EPS-AG, Lucerne, 2004).

[65] R. M. Jones et al., Proceedings of 18th International Linear Accelerator Conference (Linac 96), Geneva, Switzerland, 1996 (Report No. SLAC-PUB-7287, 1996).

[66] J. Lewandowski et al., Proceedings of 22nd International Linear Accelerator Conference (LINAC 2004), Lübeck, Germany, 2004 (Report No. SLAC-PUB-11193, 2004).

[67] L. Laurent, Ph.D. thesis, University of California, Davis, 2002.

[68] International Committee for Future Accelerators (ITRP): http://www.ligo.caltech.edu/skammer/ITRP_Home.htm.

[69] A. Grudiev and W. Wuensch, Proceedings of the 24th Linear Accelerator Conference (LINAC08), Victoria, Canada, 2008 (Ref. [19]), THP063.

[70] A. Grudiev, S. Calatroni, and W. Wuensch [Phys. Rev. ST Accel. Beams (to be published)].

[71] T. Higo (private communication).

[72] R. Assman et al., SLAC Report No. SLAC-PUB AP-103, 1997.

[73] P. Tenenbaum, Proceedings of the 21st Particle Accelerator Conference, Knoxville, 2005 (Ref. [62]). 